\title{
Protection against malaria a real possibility
}

T he results of an early-stage clinical trial have brought closer the prospect of a vaccine to help protect infants and children from becoming infected with a parasite that causes the most deadly form of malaria, a disease that, according to the WHO, kills more than one million people every year (1).

Although the primary goal of the phase I/IIb clinical trial was to assess the safety of the vaccine (RTS,S/AS02D) in infants in Mozambique, where malaria is endemic, it was also able to provide statistically significant data regarding the clinical efficacy of the vaccine. The results, published recently in The Lancet (2), indicate that the vaccine was safe and - like the standard vaccines that protect against diphtheria, tetanus, pertussis (whooping cough), and Haemophilus influenzae type b (DTP/Hib) and polio (OPV) - only provoked local swelling and pain. In terms of clinical efficacy, the vaccine was shown to reduce by $65 \%$ the number of infections with Plasmodium falciparum, the parasite that causes the most deadly form of malaria.

Most people at risk of developing malaria live in developing countries, and although individuals of all ages can contract malaria, children under the age of two are particularly vulnerable (1). Current approaches to curbing this disease rely on early diagnosis and rapid treatment, as well as programs that aim to control transmission of the disease-causing parasite either by eliminating the mosquitoes that inoculate individuals with the parasite (indoor residual insecticide spraying) or by preventing individuals from being bitten by the mosquitoes (insecticide-treated bed nets). However, there is currently only one group of drugs that are effective treatments for malaria artemisinin-based combination therapies (ACTs) - as many cases of the disease are now caused by $P$. falciparum that is resistant to former antimalarial drugs such as chloroquine and sulfadoxine-pyrimethamine. Further, there are no effective alternatives to artemisinins on the market or nearing the end of development, meaning that additional approaches, such as vaccines, to reducing the burden of disease and death caused by malaria are needed.

The new vaccine, RTS,S/AS02D, was developed by GlaxoSmithKline (GSK) and comprises RTS,S, a hybrid recombinant protein consisting of two portions of the $P$. falciparum circumsporozoite protein fused to a portion of the hepatitis B virus $S$ antigen, and AS02D, an adjuvant that boosts the immune response to RTS,S. In the trial it was shown that three doses of RTS,S/ AS02D, given at 10,14, and 18 weeks of age, primed the immune system of infants to produce large amounts of antibodies specific for the circumsporozoite protein (2). These antibodies stop P. falciparum from entering the cells of the liver, which is where the parasite multiplies, and are thought to provide the protection against malaria engendered by the vaccine.

Although the vaccine was developed by GSK, the trial was carried out in collaboration with a number of other organizations. In particular, financial support was provided by the PATH Malaria Vaccine Initia-

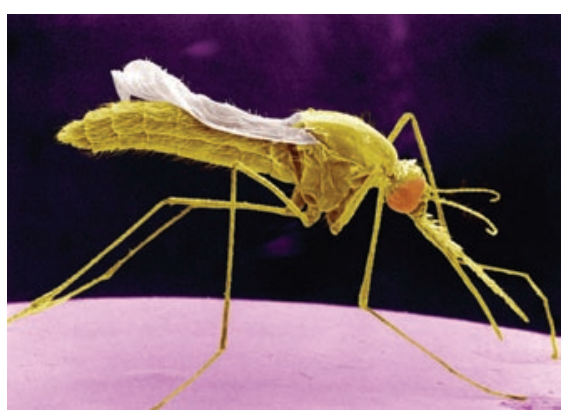

The parasites that cause malaria are transmitted to people through the bite of an infected mosquito. Image credit: Photo Researchers Inc.

tive, a malaria-focused vaccine development program established by a grant from the Bill and Melinda Gates Foundation, and the trial was conducted at the Centro de Investigação em Saúde de Manhiça, Mozambique, by scientists from the University of Barcelona. Those conducting the trial were delighted with the encouraging results. "It's very good news," said Pedro Alonso of the University of Barcelona, Spain, who led the trial (3). However, the study was relatively small including 214 infants, of whom half received RTS,S/AS02D and half a hepatitis B vaccine - so Christian Loucq (Director of the PATH Malaria Vaccine Initiative) has said that "Next year we will start a trial in 16,000 kids in 10 sites in seven African countries" (3). In addition, Brian Greenwood, an expert in malaria prevention and director of the Gates
Malaria Partnership at the London School of Hygiene and Tropical Medicine, told the JCI that studies showing that RTS,S/AS02D can be given at the same time as other infant vaccines without having an adverse effect on the responses they generate would be something that would greatly facilitate the deployment of the vaccine.

These encouraging results came as Bill and Melinda Gates, speaking at the Bill \& Melinda Gates Foundation Malaria Forum in Seattle on October 17, called on scientists and policy makers to seek to eradicate malaria rather than simply keep it under control $(4,5)$. They promised that the foundation would continue to support efforts to achieve this goal but highlighted the need for more public-private partnerships, such as the one between GSK and the PATH Malaria Vaccine Initiative, to fund the development of additional vaccines and drugs to prevent and treat malaria.

Despite the immense boost that the RTS,S/AS02D trial has given researchers in the field of malaria vaccine research, as Greenwood pointed out to the JCI, "RTS,S should be considered only as a first-generation vaccine," and even JP Garnier, chief executive officer of GSK, notes that the vaccine "will need to be deployed in combination with existing prevention and treatment tools, including bed nets and spraying" (6).

\section{Karen Honey}

1. WHO. 2007. 10 facts on malaria. http://www.who. int/features/factfiles/malaria/en/index.html.

2. Aponte, J.J., et al. 2007. Safety of the RTS,S/AS02D candidate malaria vaccine in infants living in a highly endemic area of Mozambique: a double blind randomised controlled phase I/IIb trial. Lancet. In press.

3. NewScientist.com news service. 2007 October 17. Malaria vaccine protects infants against infection. New Scientist. http://www.newscientist.com/article/mg19626263.200-malaria-vaccine-protectsinfants-against-infection.html.

4. Gates, M.F. 2007 October 17. Malaria forum keynote address. Seattle, Washington, USA. http:// www.gatesfoundation.org/MediaCenter/Speeches/ Co-ChairSpeeches/MelindaSpeeches/MFGSpeechMalariaForum-071017.htm?version = print .

5. Gates, B. 2007 October 17. Malaria forum keynote address. Seattle, Washington, USA. http://www. gatesfoundation.org/MediaCenter/Speeches/CoChairSpeeches/BillgSpeeches/BGSpeechMalariaForum-071017.htm?version $=$ print.

6. Garnier, J.P. 2007 October 18. JP Garnier: access to medicine in the developing world. Times Online. http://business.timesonline.co.uk/tol/business/ related_reports/business_ideas/article2687946. ece?token $=$ null $\&$ offset $=0$. 